\title{
Correlation Inequalities and the Decay of Correlations in Ferromagnets ${ }^{\star}$
}

\author{
Barry Simon \\ Departments of Mathematics and Physics, Princeton University, Princeton, NJ 08544, USA
}

\begin{abstract}
We prove a variety of new correlation inequalities which bound intermediate distance correlations from below by long distance correlations. Typical is the following which holds for spin $1 / 2$ nearest neighbor Ising ferromagnets :
\end{abstract}

$$
\left\langle s_{\alpha} s_{\gamma}\right\rangle \leqq \sum_{\delta \in B}\left\langle s_{\alpha} s_{\delta}\right\rangle\left\langle s_{\delta} s_{\gamma}\right\rangle
$$

where $B$ is any subset of the lattice whose removal divides the lattice into pieces with $\alpha, \gamma$ in distinct components. We describe various applications, e.g. the above inequality implies the critical exponent inequality $\eta<1$.

\section{Introduction}

This paper originated in my attempt to understand some results announced by Dobrushin [7] in the summer of 1979. Dobrushin considers a class of model systems including lattice gas models. Given two bounded regions $\Lambda \subset \Lambda^{\prime}$, he considers the variation of a Gibbs state restricted to $\Lambda$ as spins exterior to $\Lambda^{\prime}$ are varied. Among other results, he proved that if this dependence falls off as the inverse of a sufficiently high power of $d\left(\partial \Lambda, \partial \Lambda^{\prime}\right)$, then it automatically falls exponentially. It was this kind of result that I wanted to understand. We will deal with the related result that falloff of the two point function at a sufficiently fast inverse power rate implies exponential decay. We will accomplish this by proving various new correlation inequalities. Typical of the results we will prove is:

Theorem 1.1. Let $\left\langle\sigma_{\alpha} \sigma_{\gamma}\right\rangle$ denote the two point function of a spin $1 / 2$ nearest neighbor (infinite volume, free boundary condition) Ising ferromagnet at at some fixed temperature. Fix $\alpha, \gamma$ and $B$, a set of spins whose removal breaks the lattice in such $a$ way that $\alpha$ and $\gamma$ lie in distinct components. Then:

$$
\left\langle\sigma_{\alpha} \sigma_{\gamma}\right\rangle \leqq \sum_{\delta \in B}\left\langle\sigma_{\alpha} \sigma_{\delta}\right\rangle\left\langle\sigma_{\delta} \sigma_{\gamma}\right\rangle
$$

* Research partially supported by USNSF under Grant MCS-78-01885 
Let us illustrate how (1.1) implies:

Theorem 1.2. Under the hypothesis of Theorem 1.1, suppose that

$$
\left\langle\sigma_{\alpha} \sigma_{\gamma}\right\rangle \leqq C|\alpha-\gamma|^{-\mu}
$$

with $\mu+1>d$, the dimension of the lattice then

$$
\left\langle\sigma_{\alpha} \sigma_{\gamma}\right\rangle \leqq C e^{-m|\alpha-\gamma|}
$$

for some $m>0$.

Given (1.1), Theorem 1.2 clearly follows from the next result. Ideas of this genre appear to be found first in Krinsky and Emergy [27] (see also Lebowitz [29]) who obtained mass gaps from Griffiths third inequality [20] (see Sect. 4 below).

Theorem 1.3. Let $f(\alpha-\gamma)$ be a translation invariant non-negative bounded function of $\alpha, \gamma \in Z^{v}$. Suppose that for some subset $B$ of $Z^{v}$ with $B \subset\{\delta|| \delta \mid \leqq R\}$ and all $\gamma$ with $|\gamma|>R$, we have that

$$
f(\gamma) \leqq \sum_{\delta \in B} a(\delta) f(\gamma-\delta),
$$

where $0 \leqq a(\delta)$ and

$$
\sum_{\delta \in B} a(\delta) \equiv A_{0}<1 .
$$

Then

$$
|f(\gamma)| \leqq\|f\|_{\infty} \exp \left(-m_{0} g(\gamma)\right),
$$

where

$$
\begin{aligned}
\|f\|_{\infty} & =\sup _{\gamma}|f(\gamma)| \\
g(\gamma) & =R[|\gamma| / R]
\end{aligned}
$$

with $[x]=$ integral part of $x$, and

$$
m_{0}=-R^{-1} \ln A_{0} .
$$

In particular, the "mass gap" of $f$

$$
m=\liminf _{|\gamma| \rightarrow \infty}\left[-|\gamma|^{-1} \ln f(\gamma)\right]
$$

is at least $m$, and, thus it is positive.

Proof. Let $n=[|\gamma| / R]$. Then, by the translation invariance (12) may be interated $n$ times to yield

$$
|f(\gamma)| \leqq A_{0}^{n}\|f\|_{\infty}
$$

which is (1.4).

Remark. Translation invariance is inessential. All that is needed is that

$$
f(\alpha, \gamma) \leqq \sum_{\delta \in B} a(\delta) f(\alpha+\delta, \gamma)
$$


While, Theorem 1.3 and its proof are the easiest way to get a mass gap from (1.3), there are methods of getting better bounds than that given by (1.5). These are based on the following abstraction of the Krinsky and Emery [27] argument:

Theorem 1.4. Let $f$ obey (1.7) for $|\alpha-\gamma| \geqq R$. Suppose that (1.3) holds and that

$$
\left\|f_{\gamma}\right\|_{\infty} \equiv \sup _{\alpha}|f(\alpha, \gamma)|<\infty
$$

for some fixed $\gamma$. Then

(i) the matrix $A$ given by

$$
\begin{aligned}
& A(\alpha, \gamma)=a(\alpha-\gamma) \text { if } \quad \alpha-\gamma \in B \\
& =0 \quad \text { if } \quad \alpha-\gamma \notin B
\end{aligned}
$$

obeys $(1-A)$ is invertible as a map on $\ell^{\infty}$.

(ii) $|f(\alpha, \gamma)| \leqq\left\|f_{\gamma}\right\|_{\infty} \sum_{|\delta-\gamma|<R}(1-A)^{-1}(\delta, \gamma)$.

Remark. We do not require that $B \subset\{\gamma|| \gamma \mid<R\}$. This makes the high temperature result applicable to suitable infinite range interactions.

Proof. Let $f_{\alpha} \equiv|f(\alpha, \gamma)|$ and $q_{\alpha}=\|f\|_{\infty}$ (if $|\alpha|<R$ ) and $=0$ (if $|\alpha| \geqq R$ ). Then (1.7) becomes :

$$
f_{\alpha} \leqq q_{\alpha}+\sum_{\gamma} A(\alpha, \gamma) f_{\gamma}
$$

for all $\alpha$ including $\alpha$ with $|\alpha|<R$. Now, $A$ is a bounded map on $\ell^{\infty}$ with norm $\sum_{\gamma} a(\alpha-\gamma) \equiv A_{0}<1$. Thus $1-A$ is invertible, $\left\|A^{n}\right\| \rightarrow 0$ and $(1-A)^{-1}=\sum_{n} A^{n}$. Iterating (1.8) and using $\left\|A^{n}\right\| \rightarrow 0$ we get

$$
\begin{aligned}
f_{\alpha} & \leqq \lim _{n \rightarrow \infty}\left[\sum_{\gamma}\left(1+A+\ldots+A^{n}\right)(\alpha, \gamma) q_{\gamma}+\sum_{\gamma} A^{n+1}(\alpha, \gamma) f_{\gamma}\right] \\
& =\sum_{\gamma}(1-A)^{-1}(\alpha, \gamma) q_{\gamma}
\end{aligned}
$$

which proves (ii).

The following when used in conjunction with Griffiths third inequality allows one to recover the same mass falloff as Gross [23] obtains with Dobrushin uniqueness methods (but Gross' results hold in much greater generality).

Theorem 1.5. Let $\varrho$ be a metric on $Z^{v}$ with

$$
\sup _{\alpha}\left[\sum_{\gamma} e^{\varrho(\alpha, \gamma)} A(\alpha, \gamma)\right] \equiv A_{\varrho}<1 \text {. }
$$

Then

$$
\sup _{\alpha}\left[\sum_{\gamma} e^{\varrho(\alpha, \gamma)}(1-A)^{-1}(\alpha, \gamma)\right] \leqq\left(1-A_{\varrho}\right)^{-1} .
$$

Proof. By induction and the triangle inequality

$$
\sup _{\alpha}\left[\sum_{\gamma} e^{\varrho(\alpha, \gamma)} A^{n}(\alpha, \gamma)\right] \leqq A_{\varrho}^{n}
$$


The strongest results on the rate of exponential falloff are obtained by going to Fourier transforms; by Payley-Wiener the rate of exponential falloff of $(1-A)^{-1}(\alpha-\gamma)$ is related to the region of analyticity of the Fourier transform. If $a$ has bounded support, this is determined by the point in momentum space closest to 0 with $a(k)=1$. We illustrate with an example:

Example. In the two dimensional nearest neighbor Ising ferromagnet, (1.2) holds with $\left\langle\sigma_{\alpha} \sigma_{\gamma}\right\rangle=f(\alpha-\gamma)$ and

$$
\begin{aligned}
a(\delta) & =\tanh \beta & & |\delta|=1 \\
& =0 & & |\delta| \neq 1
\end{aligned}
$$

by Griffiths third inequality [20]. We might define $\varrho$ by $\varrho(\alpha, \beta)=\left|\alpha_{1}-\beta_{1}\right|$. Using any of these ideas, we get a mass gap if $4 \tanh \beta<1$. The mass gap using Fourier transform is determined by

$$
1 / 2(1+2 \cosh m)=(4 \tanh \beta)^{-1}
$$

and that by Theorems 1.4 and 1.5 by

$$
1 / 2\left(1+e^{m}\right)=(4 \tanh \beta)^{-1}
$$

which is clearly worse.

The contents of the remainder of this paper are as follows : in Sect. 2, we prove the inequalities (1.1) and generalizations for spin 1/2 Ising ferromagnets. In Sect. 3, we prove related inequalities for fairly general one component models by using Lebowitz' inequality. Since (1.1) is a kind of generalized third Griffiths inequality and since Dreisler et al. [10] have used Lebowitz' inequality to extend a consequence of Griffiths third inequality (absence of spontaneous magnetization at large $T$ ), it is not surprising that Lebowitz inequality is useful here. In Sects. 4 and 5 we apply the inequalities of Sects. 2 and 3 and also some extensions of Lieb which are described in Sect. 2 and proven in on accompanying paper of Lieb [30]. We note that in a second accompanying paper, Aizenman and Simon [2] describe certain extensions to multicomponent rotors.

The methods of Theorems $1.2-1.5$ provide a natural approach to exponential falloff. Two important open questions are the following: (1) Can one recover the results on mass gap in weak coupling $\left(\phi^{4}\right)_{2}$ obtained by Glimm et al. $[16,17]$ in a simpler way by using these methods? (2) Does this method provide a useful approach to the falloff of non-relativistic, especially atomic, wave functions (see $[33,5,1])$ ?

Before closing this introduction, we should note that in models with reflection positivity (see [11] and references therein) the inequalities of this paper are often complementary to the consequences of reflection positivity. For example, in a nearest neighbor simple cubic spin $1 / 2$ ferromagnetic Ising model, with $f(\boldsymbol{\alpha})=\left\langle\sigma_{\alpha} \sigma_{\mathbf{0}}\right\rangle$, reflection positivity implies that

$$
f\left(\alpha_{1}, 0, \ldots, 0\right) \leqq e^{-m\left|\alpha_{1}\right|}
$$

while the methods of this paper imply (see Sect. 5):

$$
\sum_{\alpha_{2}, \ldots, \alpha_{v}} f\left(\alpha_{1}, \ldots, \alpha_{v}\right) \geqq e^{-m\left|\alpha_{1}\right|} .
$$


I am especially grateful to R. Israel whose remarks on Dobrushin's work put me on the right track towards (1.1). Since then, I have benefited from discussions with M. Aizenman, J. Bricmont, J. Lebowitz, E. Lieb, A. Sokal, and T. Spencer.

\section{Graphical Methods}

In this section, we will prove (1.1) and various generalizations using graphical methods. Such methods were introduced by Griffiths in his original series [18-20] and extended by Kelley and Sherman [25], Griffiths et al. [22], and Newman [32]. In particular, our proof has elements in common with Griffiths' proof of his third inequality [20] and with Newman's proff of his inequality [32]. Our basic presentation of the combinatories follows Sylvester [34].

We begin with some notation. We have a finite set of spins, $\alpha, \gamma, \delta, \ldots$ and a family of "allowed" pairs $\ell=(\alpha, \gamma), \ldots$ which will be the pairs allowed to interact in the basic Hamiltonian.

Definition. Given two spins $\alpha, \gamma$, a path from $\alpha$ to $\gamma$ is a sequence $\alpha_{0}, \ldots, \alpha_{n}$ of spins with $\alpha_{0}=\alpha, \alpha_{n}=\gamma$ and each pair $\left(\alpha_{i}, \alpha_{i+1}\right)$ an allowed pair.

Definition. A set of spins $B$ is said to separate $\alpha$ and $\gamma$ if every path from $\alpha$ to $\gamma$ includes some spin in $B$ as an intermediate spin.

Definition. A graph, $\Gamma$, is a numbered set of allowed pairs with a given pair allowed to be repeated. $n_{\ell}$ is the number of times a given pair $\ell$, occurs. The boundary $\partial \Gamma$, of a graph is the set of those $\alpha$ with $\sum_{\alpha \in \ell} n_{\ell}$ an odd integer. Given an analytic function, $f$, of variables $J_{\ell}$, one for each allowed $\ell$, we let $\partial^{\Gamma} f$ denote $\partial^{\Sigma n_{\ell}} / \prod_{\ell} \partial \mathbf{J}_{\ell}^{n_{\ell}}$ f evaluated at all $J_{\ell}=0$.

Definition. Let $\Gamma$ and $G$ be graphs. We say that $G$ is a subgraph of $\Gamma$ and write $G \subset \Gamma$, if $G$ is a subset of $\Gamma$. If $H=\Gamma \backslash G$, we write $\Gamma=G \oplus H$. The symbol, $\sum_{G \oplus H=\Gamma}$, stands for the sum over all subgraphs $G$ of $\Gamma$.

Lebnitz' rule says that

$$
\partial^{\Gamma}(f g)=\sum_{G \oplus H=\Gamma}\left(\partial^{G} f\right)\left(\partial^{H} g\right) .
$$

Remark. $\partial^{G}$ only depends on the $n_{\ell}(G)$. The labelling of the lines of $\Gamma$, means that the number of $G \subset \Gamma$ with $n_{\ell}(G)=m_{\ell}$ is $\prod_{\ell}\left(\begin{array}{c}n_{\ell}(\Gamma) \\ m_{\ell}\end{array}\right)$ yielding the requisite combinatorial factors in Lebnitz' rule.

Definition. Given two spins $\alpha, \gamma$ and a set $B$ separating $\alpha$ and $\gamma$, we define the $\alpha$-side of $B$ as follows. Consider all paths from $\alpha$ to $\gamma$ and look at the segment of the path between $\alpha$ and the first hitting of $B$. The family of all links in all these segments is the $\alpha$-side of $B$. 
Thus, by construction, the $\alpha$-side of $B$ never includes any links between spins in $B$. If $B$ consists of those spins linked to $\alpha$ by a single allowed pair, then the $\alpha$ side of $B$ consists precisely of the pairs which include $\alpha$.

Theorem 1.1 is a special case of the following theorem:

Theorem 2.1. Let $H=-\sum_{\ell=(\alpha \gamma)} J_{\ell} \sigma_{\alpha} \sigma_{\gamma}$ with $\sigma_{\alpha}$ an Ising spin. Let $\langle\cdot\rangle_{0}=\sum_{\sigma_{\alpha}= \pm 1}$, as usual, and

$$
\langle\cdot\rangle=\left\langle\cdot e^{-H}\right\rangle_{0} / Z ; \quad Z=\left\langle e^{-H}\right\rangle_{0} .
$$

Suppose $J_{\ell} \geqq 0$ for all $\ell$ let $\alpha, \gamma$ be two fixed spins and let $B$ separate $\alpha$ and $\gamma$. Then:

$$
\left\langle\sigma_{\alpha} \sigma_{\gamma}\right\rangle \leqq \sum_{\delta \in B}\left\langle\sigma_{\alpha} \sigma_{\delta}\right\rangle\left\langle\sigma_{\delta} \sigma_{\gamma}\right\rangle
$$

Proof. Let

$$
f\left(J_{\ell}\right) \equiv\left[\sum_{\delta \in B}\left\langle\sigma_{\alpha} \sigma_{\delta} e^{-H}\right\rangle_{0}\left\langle\sigma_{\delta} \sigma_{\gamma} e^{-H}\right\rangle_{0}\right]-\left\langle e^{-H}\right\rangle_{0}\left\langle\sigma_{\alpha} \sigma_{\gamma} e^{-H}\right\rangle_{0} .
$$

Since $f$ is analytic, it suffices to show that

$$
\partial^{\Gamma} f \geqq 0
$$

for all graphs, $\Gamma$. If $\partial \Gamma \neq\{\alpha, \gamma\}$, it is easy to see that $\partial^{\Gamma} f=0$ since for $\delta \neq \lambda$ :

$$
\partial^{G}\left[\left\langle\sigma_{\delta} \sigma_{\lambda} e^{-H}\right\rangle_{0}\right]=\delta_{\partial G,\{\delta, \lambda\}}
$$

with $\delta$., the Kronceker $\delta$ function on sets of spins.

Thus, consider a fixed graph $\Gamma$ with $\partial \Gamma=\{\alpha, \gamma\}$. An easy inductive argument shows there exists a path $P$, from $\alpha$ to $\gamma$ which is a subgraph of $\Gamma$ (although the numbering of $P$ as a subset of $\Gamma$ and the numbering as a path may be distinct). Let $\delta_{0} \in B$ be such that $\delta_{0}$ is an intermediate spin on $P$. Let $P_{0}$ be the segment of $P$ from $\alpha$ to $\delta_{0}$. We will prove that

$$
\partial^{T}\left[\left\langle\sigma_{\alpha} \sigma_{\delta_{0}} e^{-\boldsymbol{H}}\right\rangle_{0}\left\langle\sigma_{\gamma} \sigma_{\delta_{0}} e^{-H}\right\rangle_{0}-\left\langle e^{-H}\right\rangle_{0}\left\langle\sigma_{\alpha} \sigma_{\gamma} e^{-H}\right\rangle_{0}\right]=0
$$

so that (2.2) follows from $\partial^{\Gamma}\left(\left\langle\sigma_{\alpha} \sigma_{\delta} e^{-H}\right\rangle_{0}\left\langle\sigma_{\delta} \sigma_{\gamma} e^{-H}\right\rangle_{0}\right) \geqq 0$ which is a consequence of (2.1), (2.3). By this formula,

$$
\text { LHS of }(2.4)=\sum_{G_{1} \oplus G_{2}=\Gamma} \delta_{\partial G_{1},\left\{\alpha, \delta_{0}\right\}} \delta_{\partial G_{2},\left\{\delta_{0}, \gamma\right\}}-\sum_{H_{1} \oplus H_{2}=\Gamma} \delta_{\partial H_{1}, \emptyset} \delta_{\partial H_{2},\{\alpha, \gamma\}} .
$$

We claim that the formulae, $H_{1}=G_{1} \Delta P_{0}, H_{2}=G_{2} \Delta P_{0}(\Delta=$ symmetric difference of subsets) set up a one-one correspondence between $G_{1} \oplus G_{2}=\Gamma$ and $H_{1} \oplus H_{2}=\Gamma$ so that, under this correspondence, there is a term-by-term cancelation between the two sums in the last formulae. This proves (2.4) and so (2.2).

Lieb [30] has extended the proof above to show

Theorem 2.2 [30]. Under the hypotheses of Theorem 2.1, let $L$ be the $\alpha$ side of $B$ and let $H^{1}=-\sum_{\ell \in L} J_{\ell} \sigma_{\alpha} \sigma_{\gamma},\langle-\rangle^{1}=\left\langle-e^{-H^{1}}\right\rangle_{0} / Z^{1}$ etc. Then

$$
\left\langle\sigma_{\alpha} \sigma_{\gamma}\right\rangle \leqq \sum_{\delta \in B}\left\langle\sigma_{\alpha} \sigma_{\delta}\right\rangle^{1}\left\langle\sigma_{\delta} \sigma_{\gamma}\right\rangle
$$


Remarks. 1. By Griffiths' second inequality $\left\langle\sigma_{\alpha} \sigma_{\delta}\right\rangle^{1} \leqq\left\langle\sigma_{\alpha} \sigma_{\delta}\right\rangle$, so Lieb's result is always an improvement on Theorem 2.1.

2. If $B$ consists of those spins directly linked to $\alpha$, then $\left\langle\sigma_{\alpha} \sigma_{\delta}\right\rangle^{1}=\tanh J_{\ell}$ so that Theorem 2.2 includes Griffiths' third inequality as a special case.

The following pair of results have proofs essentially identical to that of Theorem 2.1.

Theorem 2.3. Let $H,\langle-\rangle$ be as in Theorem 2.1. Let $A, B, C$ be three sets, so that for any $\alpha \in A, \gamma \in C, B$ separates $\alpha$ and $\gamma$. Let $\sigma^{D}=\prod_{\alpha \in D} \sigma_{\alpha}$. Then

$$
\left\langle\sigma^{A} \sigma^{C}\right\rangle \leqq \sum_{D \subset B, \#(D) \leqq \#(A)}\left\langle\sigma^{A} \sigma^{D}\right\rangle\left\langle\sigma^{D} \sigma^{C}\right\rangle .
$$

Remarks. 1. Theorem 2.2 has an analog of this form.

2. Given any graph $G$, with $\partial G=A \cup C$, we find easily a subgraph $P_{0}=$ union of paths with $\partial P_{0}=A \cup D$, where each path has either both ends in $A$ or one in $A$ and one in $D$. Note if $A$ is even we must allow $D=\emptyset$.

To extend to multispin interactions, we introduce the following extended notation: (1) We have a set $\mathscr{S}=\{\alpha, \gamma, \ldots\}$ of spins and an allowed family $\mathscr{A}$ of subsets of $\mathscr{S}$. The Hamiltonian will have the form

$$
-H=\sum_{A \in \mathscr{A}} J_{A} \sigma^{A} ; \quad J_{A} \geqq 0 .
$$

(2) Graphs are just numbered subsets of $\mathscr{A}$ with repetitions allowed.

(3) Given subsets, $A, C \in S$, a "path" is a graph $\Gamma$ with $\partial \Gamma=A \cup C$.

(4) We say that $\mathscr{B} \subset \mathscr{S}$ separates $A$ and $C$ if any path, $\Gamma$, from $A$ to $C$ can be written $\Gamma=P_{1} \oplus P_{2}$ with $\partial P_{1}=A \cup B, \partial P_{2}=B \cup C$ for some $B \in \mathscr{B}$.

(5) The set of all $D \in \mathscr{A}$ which arises in the minimal $P_{1}$ 's which occur in (4) are the $A$-side of $\mathscr{B}$.

Theorem 2.4. Under the above notations with $H$ given by (2.5) and $\mathscr{B}$ separating $A$ and $C$, we have that

$$
\left\langle\sigma^{A} \sigma^{C}\right\rangle \leqq \sum_{B \in \mathscr{B}}\left\langle\sigma^{A} \sigma^{B}\right\rangle\left\langle\sigma^{B} \sigma^{C}\right\rangle .
$$

Remark. In the above, one can replace $\left\langle\sigma^{A} \sigma^{B}\right\rangle$ by $\left\langle\sigma^{A} \sigma^{B}\right\rangle^{1}$. In particular, if $A=\{\alpha\}$, if $\mathscr{B}=\{B \cup \alpha / B \cup(\alpha) \in \mathscr{A}\}$, and if any graph of $\{B \cup\{\alpha\} \mid B \in \mathscr{B}\}$ with empty boundary has each $n(B \cup \alpha)$ even, then

$$
\left\langle\sigma_{\alpha} \sigma^{C}\right\rangle \leqq \sum_{B \in \mathscr{B}}\left(\tanh J_{B \cup \alpha}\right)\left\langle\sigma^{B} \sigma^{C}\right\rangle
$$

which is a generalized Griffiths' third inequality [20,35].

By Griffiths' trick [21], one immediately has :

Theorem 2.5. Let each $\sigma_{\alpha}$ take the values $-2 S,-2 S+2, \ldots, 2 S$ and let $H,\langle\rangle,\langle\rangle_{0}$, be as in Theorem 2.1, except that $\langle\cdot\rangle_{0}=\sum_{\sigma_{\alpha}=-2 S, \ldots, 2 S} \cdot$ Then

$$
\left\langle\sigma_{\alpha} \sigma_{\gamma}\right\rangle \leqq \sum_{\delta \in B}\left\langle\sigma_{\alpha} \sigma_{\delta}\right\rangle\left\langle\sigma_{\delta} \sigma_{\gamma}\right\rangle
$$


Proof. By Griffiths' method, we can find a spin $1 / 2$ system, with spins $\left\{S_{\alpha, 1}, \ldots, S_{\alpha k}\right\}$ with $k=2 S$ so that

$$
\left\langle\sigma_{\lambda} \sigma_{\mu}\right\rangle=\sum_{p q}\left\langle S_{\lambda p} S_{\lambda q}\right\rangle_{a}
$$

and $\langle-\rangle_{a}$ an analog ferromagnetic coupling with couplings between $S_{\lambda p}, S_{\mu q}$ with $(\lambda \mu)$ an allowed pair and $S_{\lambda p}, S_{\lambda q}$. If we let $\mathscr{B}_{a}=\left\{S_{\lambda p} \mid \lambda \in \mathscr{B}\right\}$, we have

$$
\begin{aligned}
\left\langle\sigma_{\alpha} \sigma_{\gamma}\right\rangle & =\sum_{p q}\left\langle S_{\alpha p} S_{\gamma q}\right\rangle_{a} \\
& \leqq \sum_{p, q, \delta \in B, r}\left\langle S_{\alpha p} S_{\delta r}\right\rangle_{a}\left\langle S_{\delta r} S_{\gamma q}\right\rangle_{a} \\
& \leqq \sum_{\delta \in B}\left\langle\sigma_{\alpha} \sigma_{\delta}\right\rangle\left\langle\sigma_{\delta} \sigma_{\gamma}\right\rangle
\end{aligned}
$$

when we have used Theorem 2.1 on the analog system.

Remark. This result is not in a suitable form for going to the $S \rightarrow \infty$ limit. In (2.6), we have thrown something away since $\left\langle S_{\alpha p} S_{\delta r}\right\rangle_{a}\left\langle S_{\delta t} S_{\gamma q}\right\rangle_{a}$ with $r \neq t$ have been added. Some of this may be recovered [e.g. if $S=1$, we could place a $1 / 2$ in front of (2.6)] but not enough to go to the $S \rightarrow \infty$ limit. In the next section we will obtain an inequality in the $S=\infty$ which, while different from (1.1), is sufficient for most application (namely those not using Lieb's improvement. Theorem 2.2).

\section{Lebowitz Inequality Methods}

Definition. Let $d \mu$ be a measure on $(-\infty, \infty)$. We say it is a Lebowitz measure, if it is even and every pair interacting ferromagnet with a priori distributions $d \mu$ obeys Lebowitz inequality [28]:

$$
\begin{aligned}
\left\langle\sigma_{\alpha} \sigma_{\beta} \sigma_{\gamma} \sigma_{\delta}\right\rangle \leqq & \left\langle\sigma_{\alpha} \sigma_{\beta}\right\rangle\left\langle\sigma_{\gamma} \sigma_{\delta}\right\rangle+\left\langle\sigma_{\alpha} \sigma_{\gamma}\right\rangle\left\langle\sigma_{\beta} \sigma_{\delta}\right\rangle \\
& +\left\langle\sigma_{\alpha} \sigma_{\delta}\right\rangle\left\langle\sigma_{\beta} \sigma_{\gamma}\right\rangle .
\end{aligned}
$$

Remark. By results of Ellis et al. [9], if $d \mu(x)=e^{-v(x)} d x$ with $v$ even, $C^{1}$, and $v^{\prime}(x)$ convex on $(0, \infty)$, then $d \mu$ is a Lebowitz measure; this includes $\phi^{4}$ lattice field theories and (as a limit) spins uniformly distribution on $[-1,1]$.

Theorem 3.1. Let $\langle\cdot\rangle$ be the expectation for a pair interacting ferromagnet with an a priori single spin measure which is a Lebowitz measure. Suppose the magnet is in zero external field in finite volume (or a limit of such states). Let $A, C$ be two disjoint subsets whose union is all spins.

Let $\alpha \in A, \gamma \in C$. Then

$$
\left\langle\sigma_{\alpha} \sigma_{\gamma}\right\rangle \leqq \sum_{\substack{\delta \in A \\ \mu \in C}} J_{\delta \mu}\left[\left\langle\sigma_{\alpha} \sigma_{\delta}\right\rangle\left\langle\sigma_{\mu} \sigma_{\gamma}\right\rangle+\left\langle\sigma_{\alpha} \sigma_{\mu}\right\rangle\left\langle\sigma_{\delta} \sigma_{\gamma}\right\rangle\right] .
$$

Remark. (1.1) is not suitable for general single spin distributions, since it is not invariant under changing $\sigma_{\alpha}$ to $S \sigma_{\alpha}$. Since this change replaces $J_{\alpha \mu}$ by $S^{-2} J_{\alpha \mu},(3.2)$ is invariant under such changes. Notice the other differences between (3.2) and (1.1); the latter is an expansion in "intermediate sites", the former in "intermediate bonds". 
Proof. Let $\langle\cdot\rangle_{\varepsilon}$ denote the spin system obtained by replacing each $J_{\delta \mu}$ with $\delta \in A$, $\mu \in C$ by $\varepsilon J_{\delta \mu}$.

Then $\left\langle\sigma_{\alpha} \sigma_{\gamma}\right\rangle_{\varepsilon=0}=0$ so

$$
\left\langle\sigma_{\alpha} \sigma_{\gamma}\right\rangle=\int_{0}^{1} \frac{d}{d \varepsilon}\left[\left\langle\sigma_{\alpha} \sigma_{\gamma}\right\rangle_{\varepsilon}\right] d \varepsilon .
$$

Now, by Lebowitz inequality

$$
\begin{aligned}
\frac{d}{d \varepsilon}\left\langle\sigma_{\alpha} \sigma_{\gamma}\right\rangle_{\varepsilon} & =\sum_{\substack{\delta \in A \\
\mu \in C}} J_{\delta \mu}\left[\left\langle\sigma_{\alpha} \sigma_{\gamma} \sigma_{\mu} \sigma_{\delta}\right\rangle_{\varepsilon}-\left\langle\sigma_{\alpha} \sigma_{\gamma}\right\rangle_{\varepsilon}\left\langle\sigma_{\mu} \sigma_{\delta}\right\rangle_{\varepsilon}\right] \\
& \leqq \sum_{\substack{\delta \in A \\
\mu \in C}} J_{\delta \mu}\left[\left\langle\sigma_{\alpha} \sigma_{\mu}\right\rangle_{\varepsilon}\left\langle\sigma_{\gamma} \sigma_{\delta}\right\rangle_{\varepsilon}+\left\langle\sigma_{\alpha} \sigma_{\delta}\right\rangle_{\varepsilon}\left\langle\sigma_{\gamma} \sigma_{\mu}\right\rangle_{\varepsilon}\right] \\
& \leqq \text { rhs of (3.2) }
\end{aligned}
$$

by the second GKS inequality which holds for even $\mu[13,24]$.

The above proof depended on two things:

(i) Lebowitz inequality which is known to hold for $N$-component molds with $N=2,3,4[4,8]$.

(ii) The GKS II inequality which holds for two component models [13]. We therefore have:

Theorem 3.2. (3.2) holds when $\sigma_{\alpha}$ is the first component of two component isotropically coupling (plane rotor) ferromagnets.

In [2], Aizenman and Simon prove a strictly stronger inequality than (3.2) for $N=2,3,4$ component models.

\section{Bounds on Critical Temperatures}

In this section, we want to apply the methods of this paper to obtain upper bounds on $T_{C}$, the critical temperatures, defined to be that temperature at which the two point function no longer falls exponentially. While we will state results for spin $1 / 2$ Ising (or plane rotor) ferromagnetic models, there are automatically results for general interactions, since $\left|\left\langle\sigma_{\alpha} \sigma_{p}\right\rangle_{J}\right| \leqq\left\langle\sigma_{\alpha} \sigma_{p}\right\rangle_{|J|}$, i.e. correlations increase if $J_{\alpha p}$ is replaced by $\left|J_{\alpha p}\right|$. Also, since correlations go up if an even single spin measure on $[-S, S]$ is replaced by $\delta(\sigma-S)+\delta(\sigma+S)$ [36], we get bounds for general single spin meásures.

We begin by recalling the Krinsky-Emery result [27].

Theorem 4.1. [27]. If $H=-\sum J_{\alpha \gamma} \sigma_{\alpha} \sigma_{\gamma}, J_{\alpha \gamma}=J_{\alpha-\gamma} \geqq 0$, if $\langle\cdot\rangle$ is the infinite volume free b.c. state, if

$$
\sum \tanh J_{\alpha 0}<1
$$

and if $\left\{\alpha \mid J_{\alpha 0} \neq 0\right\}$ is bounded, then

$$
\left\langle\sigma_{\alpha} \sigma_{\gamma}\right\rangle \leqq e^{-m|\alpha-\gamma|}
$$

for some $m>0$. 
Proof. By Griffiths' third inequality

$$
\left\langle\sigma_{0} \sigma_{\gamma}\right\rangle \leqq \sum_{\alpha}\left(\tanh J_{0 \alpha}\right)\left\langle\sigma_{\alpha} \sigma_{\gamma}\right\rangle .
$$

Now use Theorem 1.3.

In [2] Aizenman and Simon prove new inequalities and then use these ideas to prove the following "mean-field" bounds:

Theorem 4.2 [2]. If $\boldsymbol{\sigma}_{\alpha}$ are $N$ component unit spins, if $H=-\sum J_{\alpha \gamma} \boldsymbol{\sigma}_{\alpha} \boldsymbol{\sigma}_{\gamma}$, $J_{\alpha \gamma}=J_{\alpha-\gamma} \geqq 0$, if $\langle\cdot\rangle$ is an infinite volume periodic b.c. state, if

$$
\sum J_{\alpha 0}<N
$$

and if $\left\{\alpha \mid J_{\alpha 0} \neq 0\right\}$ is bounded, then

$$
\left\langle\boldsymbol{\sigma}_{\alpha} \cdot \boldsymbol{\sigma}_{\gamma}\right\rangle \leqq e^{-m|\alpha-\gamma|}
$$

for some $m>0$.

In particular, if we look at two-dimensional, two component spins with coupling $\beta$, we get from (4.1) the bound

$$
\beta_{c} \geqq 0.5
$$

(from $4 \beta_{c} \geqq 2$ ) on the putative Kosterlitz-Thouless transition. Unfortunately, (4.2) is off by more than a factor of two from the current best numerical bounds [26] and Fröhlich and Spencer [12] have used different methods to get $\beta_{c} \geqq 0.66$ and. Aizenman and Simon [3], using still different methods, have obtained $\beta_{c} \geqq 0.88$.

One of the more exciting consequences of the ideas of this paper is the possibility of obtaining a sequence of lower bounds $\beta_{c}^{(n)}$ on $\beta_{c}$ so that each $\beta_{c}^{(n)}$ is computable by a finite algorithm while one is guaranteed that

$$
\lim _{n \rightarrow \infty} \beta_{c}^{(n)}=\beta_{c} .
$$

We describe the ideas for Ising models.

Theorem 4.3. Let $\Lambda^{(n)}$ be a sequence of increasing volumes, so that

(i) $\bigcup \Lambda^{(n)}=Z^{v}$.

(ii) Let

$\partial \Lambda=\left\{\alpha \in \Lambda \mid\right.$ some neighbor of $\alpha$ is in $\left.Z^{v} / \Lambda\right\}$.

We suppose that $\left|\partial \Lambda^{(n)}\right|$, the number of points in $\partial \Lambda^{(n)}$ is bounded by some power of $d\left(0, \partial \Lambda^{(n)}\right)$, the distance of 0 from $\partial \Lambda^{(n)}$. Define $\beta_{c}^{(n)}$ by:

$$
\sum_{\alpha \in \partial \Lambda^{(n)}}(n)\left\langle\sigma_{0} \sigma_{\alpha}\right\rangle_{\beta \varepsilon^{(n)}, \Lambda^{(n)}}=1,
$$

where $\langle\cdot\rangle_{\beta, \Lambda}$ is the free B.C. state of the nearest neighbor model at inverse temperature $\beta$ and volume $\Lambda$. Then $\beta_{c}^{(n)} \leqq \beta_{c}$ the critical temperature (defined by loss of exponential falloff) and (4.5) holds.

Remark. Since $\sum\left\langle\sigma_{0} \sigma_{\alpha}\right\rangle_{\beta, \Lambda^{(n)}}$ is strictly monotone in $\beta$ and is zero at $\beta=0$ and $\left|\partial \Lambda^{(n)}\right|$ at $\beta=\infty$, there is a unique $\beta_{c}^{(n)}$ obeying (4.6). 
Proof. By Theorem 1.1, 1.2, and 2.2, $\beta_{c}^{(n)} \leqq \beta_{c}$. Let $\beta<\beta_{c^{*}}$. Then, by definition $\left\langle\sigma_{0} \sigma_{\alpha}\right\rangle_{\beta, Z^{v}}$ has exponential falloff, so

$$
\lim _{n \rightarrow \infty} \sum_{\alpha \in \partial \Lambda^{(n)}}\left\langle\sigma_{0} \sigma_{\alpha}\right\rangle_{\beta, Z^{\nu}}=0
$$

and thus, since $\left\langle\sigma_{0} \sigma_{\alpha}\right\rangle_{\beta, \Lambda^{(n)}} \leqq\left\langle\sigma_{0} \sigma_{\alpha}\right\rangle_{\beta, Z^{\nu}}, \beta_{c}^{(n)}>\beta$ for $n$ large. This proves (4.5).

As for the choice of $\Lambda^{(n)}$, the following seems to be a particularly good choice. Let $\tilde{\Lambda}^{(n)}$ be the hypercube with volume $(2 n+1)^{v}$ centered at the origin. Let $\Lambda^{(n)}$ be the union of $\tilde{\Lambda}^{(n)}$ and its nearest neighbor (see Fig. 1 for $v=2, n=1$ so that $\left.\partial \Lambda^{(n)}=\Lambda^{(n)} \backslash \tilde{\Lambda}^{(n)}\right)$.

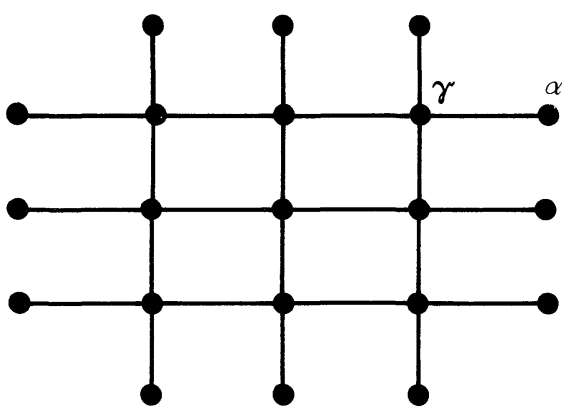

Fig. 1

Do not couple the spins in $\partial \Lambda^{(n)}$ to each other. The extra complication of the outside spins allows a rather cheap improvement since, e.g. in Fig. 1,

$$
\left\langle\sigma_{\delta} \sigma_{\alpha}\right\rangle=\tanh \beta\left\langle\sigma_{\delta} \sigma_{\gamma}\right\rangle
$$

for any $\delta \neq \alpha$. In this case, one gets

$$
\tanh \beta_{c}^{(0)}=(2 v)^{-1} \text {. }
$$

Using high temperature expansions one sees that the right side of (4.6) for the $\Lambda^{(n)}$ shown in Fig. 1 is

$$
4 a^{2} X+8 a^{3} Y / Z
$$

with

$$
\begin{aligned}
& X=1+2 a^{2}+4 a^{4}+6 a^{6}+3 a^{8}, \\
& Y=2+2 a^{2}+6 a^{4}+6 a^{6}, \\
& Z=1+4 a^{4}+4 a^{6}+7 a^{8}
\end{aligned}
$$

with $a=\tanh \beta_{c}^{(1)}$. One finds

$$
\tanh \beta_{c}^{(1)}=0.31328 \text {. }
$$

While (4.9) is an improvement on the value 0.25 of (4.8) it is disappointing how far it still is from the known exact value of $\tanh \beta_{c}^{(\infty)}=0.414$ and from the bound of Fisher [10]: $\tanh \beta_{c}^{(\infty)} \geqq 0.38$; in fact, with Fisher's method and virtually no work, one can get $\tanh \beta_{c}^{(\infty)} \geqq 0.33$ improving (4.9). Moreover, exact calculations for $n \geqq 2$ 
or $v \geqq 3 ; n \geqq 1$ seem virtually impossible. $Z$ in the above case is based on looking at 16 high temperature diagrams. For $v=2, n=2$ there are roughly 65,000 diagrams and for $v=3, n=1$ roughly 130,000 diagrams! In addition, the rate of convergence will be very slow. Roughly speaking $\beta_{c}^{(n)}$ will be about that inverse temperature at which the correlation length is $n$ so that $\beta_{c}^{(n)}-\beta_{c}^{(\infty)}$ will converge as $n$ to some negative power. Since $\beta_{c}^{(n)}$ involves roughly $a^{n}$ work, the rate of convergence is only logarithmic.

Nonetheless, we feel that it is an interesting matter of principle that one can obtain $\beta_{c}$ in an algorithmic manner. Moreover, one can modify the above and obtain bounds $\tilde{\beta}_{c}^{(n)}$ which also converges to $\beta_{c}$ and which involves somewhat less calculations. Here is one possibility which we find especially attractive: Let $m_{n}(\beta)$ be the mass gap for a $(2 n+1)^{v-1}$ transfer matrix. Determine $\widetilde{\beta}^{(n)}$ by:

$$
(2 v)\left[\tanh \tilde{\beta}^{(n)}\right] e^{-n m}\left(1-e^{-m}\right)^{-(v-1)}=1 .
$$

We claim that

Theorem 4.4. $\tilde{\beta}^{(n)} \leqq \beta_{c}$ and $\lim _{n \rightarrow \infty} \tilde{\beta}^{(n)}=\beta_{c}$.

Proof. Let us show that the left side of (4.10) dominates $\sum_{\alpha \in \partial \Lambda^{(n)}}\left\langle\sigma_{\alpha} \sigma_{0}\right\rangle$ with $\Lambda^{(n)}$ that used in the above example. This implies the inequality. The limiting statement follows as in Theorem 4.3 if one notes that $m_{n}(\beta)$ is always dominated from below by the mass gap of the infinite volume theory. Let $\gamma \in \partial \tilde{\Lambda}_{n}$ and let $\#(\gamma)$ be the number of points in $2 \Lambda_{n}$ adjacent to $\gamma$. Clearly $\#(\gamma)$ is the number of distinct faces of $\partial \Lambda_{n}$ in which $\gamma$ lies. By (4.7):

$$
\begin{aligned}
\sum_{\alpha \in \partial \Lambda^{(n)}}\left\langle\sigma_{\alpha} \sigma_{0}\right\rangle & =(\tanh \beta) \sum_{\gamma \in \partial \tilde{\Lambda}^{(n)}} \#(\gamma)\left\langle\sigma_{\gamma} \sigma_{0}\right\rangle \\
& =(2 v)(\tanh \beta) \sum_{\gamma \in \text { one face }}\left\langle\sigma_{\gamma} \sigma_{0}\right\rangle
\end{aligned}
$$

so we need to show that

$$
\sum_{\gamma \in \text { one face }}\left\langle\sigma_{\gamma} \sigma_{0}\right\rangle \leqq e^{-m n}\left(1-e^{-m}\right)^{-(v-1)} .
$$

Given the fact that the two point function in $\tilde{\Lambda}_{n}$ is bounded by that in a $(2 n+1)^{v-1} x(-\infty, \infty)$ "cylinder", (4.11) is a simple exercise in the use of transfer matrices.

As a final remark on exponential falloff at high temperatures : it is easy using the results of Sect. 3 to show that a lattice $\lambda \phi^{4}$ field theory at sufficiently large bare mass has a mass gap. However, this also follows from simple correlation inequalities.

\section{Bounds on Critical Exponents and Related Quantities}

In this final section, we want to use the basic inequalities of Sects. 2 and 3 to prove various bounds on behaviour at or near the critical point. For simplicity, we state things for nearest neighbor Ising models. Except for Theorem 5.3, which uses 
Lieb's improved inequality, one can accommodate fairly general single spin distributions via the bounds of Sect. 3. Moreover, except for the few results that require reflection positivity, general finite range ferromagnetic interactions can be accommodated also. We begin by restating Theorem 1.2:

Theorem 5.1. The critical exponent $\eta$ of the Ising model is at most 1.

Remarks. 1. $\eta$ is defined by $\left\langle\sigma_{0} \sigma_{\alpha}\right\rangle \sim C|\alpha|^{-(d-2+\eta)}$ at the critical point. Notice to define $\eta$, one needs to suppose power falloff at the critical points. The real point of our inequalities is that one can say something independent of such assumptions. If there is no mass gap, then $\sum_{\alpha \in B}\left\langle\sigma_{0} \sigma_{\alpha}\right\rangle \geqq 1$ for all "curves" $B$ surrounding 0 .

2. This improves the result $\eta<2$ of Glimm and Jaffe [15]. Their proof, unlike ours, depends on reflection positivity and so will not hold for finite range Ising models but our proof, at present, does not handle (continuum) field theories as theirs does.

The bound $\eta \leq 1$ does not imply any bound at finite distances since the constant $C$ may be small but Theorem 1.1 does:

Theorem 5.2 (uses reflection positivity). For $n \in Z$, let $f(n)=\left\langle\sigma_{(0, \ldots, 0)} \sigma_{(n, 0, \ldots, 0)}\right\rangle$. Then

(i) $m=\lim _{n \rightarrow \infty}-n^{-1} \log f(n)$ exists.

(ii) $e^{-m n} \geqq f(n) \geqq(2 d)^{-1}(2 n+1)^{-(d-1)} e^{-m n}$.

Proof. The existence of the limit and the upper bound on $f$ are consequences of reflection positivity. Let $\varrho(\alpha, \beta)=\sup _{1 \leqq i \leqq d}\left(\alpha_{i}-\beta_{i}\right)$. The method of this paper implies that

$$
\sum_{\{\alpha \mid \varrho(\alpha)=n\}}\left\langle\sigma_{\alpha} \sigma_{0}\right\rangle e^{m \varrho(\alpha)} \geqq 1 .
$$

But, by reflection positivity, the maximum $\left\langle\sigma_{\alpha} \sigma_{0}\right\rangle$ among these $\alpha$ 's is $f(n)$. Clearly there are at most $(2 n+1)^{(d-1)}(2 d)$ such $\alpha$ 's.

Using Lieb's improved version of (1.1) (Theorem 2.2), we can find a new proof of the McBryan-Rosen result [31] that the mass gap goes to zero at the critical point:

Theorem 5.3 (uses reflection positivity and Lieb's improved inequality Theorem 2.2). Consider the nearest neighbor d-dimension spin 1/2 Ising model at inverse temperature $\beta$ and let $m(\beta)$ be defined by Theorem 5.2. Suppose that $m(\beta)>0$. Then $m\left(\beta^{\prime}\right)>0$ so long as

$$
\delta \beta \equiv \beta^{\prime}-\beta<1 / 2(2 n+1)^{-2 d+1} d^{-1}(2 d)^{-1},
$$

where $n$ is the smallest integer obeying

$$
(2 d)(2 n+1)^{d-1} e^{-m n} \leqq 1 / 2 .
$$

Remark. This result implies that $m(\beta) \rightarrow 0$ as $\beta \rightarrow \beta_{c} \equiv \inf \{\beta \mid m(\beta)=0\}$. There is a critical exponent inequality implicit here which we do not make explicit since it is 
worse than that obtained using other methods $[14,15,31]$. The ideas here also imply continuity of $m(\beta)$.

Proof. By the argument in the last theorem, which uses reflection positivity,

$$
\left\langle\sigma_{\alpha} \sigma_{0}\right\rangle_{\beta, \infty} \leqq e^{-m \delta|\alpha|}
$$

where $\langle\cdot\rangle_{\beta, k}$ means the state at inverse temperature $\beta$ and in a cube, $C_{k}$, of side $2 k+1$. By Griffiths inequalities, if $\varrho(\alpha)=n$ :

$$
\left\langle\sigma_{\alpha} \sigma_{0}\right\rangle_{\beta, n} \leqq e^{-m n}
$$

Moreover:

$$
\begin{aligned}
\frac{d}{d \beta}\left\langle\sigma_{\alpha} \sigma_{0}\right\rangle & =\sum_{\gamma \delta n n \text { in } C_{n}}\left[\left\langle\sigma_{\alpha} \sigma_{0} \sigma_{\gamma} \sigma_{\delta}\right\rangle-\left\langle\sigma_{\alpha} \sigma_{0}\right\rangle\left\langle\sigma_{\gamma} \sigma_{\delta}\right\rangle\right] \\
& \leqq \sum_{\gamma \delta n n \text { in } C_{n}} 1=(d)(2 n+1)^{d}
\end{aligned}
$$

Thus

$$
\sum_{\varrho(\alpha)=n}\left\langle\sigma_{\alpha} \sigma_{0}\right\rangle_{\beta^{\prime}, n} \leqq(2 d)(2 n+1)^{d-1}\left[e^{-m n}+(\delta \beta) d(2 n+1)^{d}\right] .
$$

(5.2) implies that the first term in $[\ldots]$ contributes at most $1 / 2$ to the right side of (5.3) and then (5.1) implies that the sum is less than 1 which implies a mass gap.

The remaining bounds depend on studying the function

$$
g(n)=\sum_{\alpha_{1}=n}\left\langle\sigma_{\alpha} \sigma_{0}\right\rangle .
$$

If we had a model with interactions between spins with $\left|\alpha_{1}-\beta_{1}\right| \leqq r$, then one would replace the condition $\alpha_{1}=n$ by $\alpha_{1}=n, n+1, \ldots, n+r-1$. We begin by noting:

Theorem 5.4. Let $m$ be given by Theorem 5.2. Then if $m>0$, the sum in (5.4) exists and

$$
\lim _{n \rightarrow \alpha}-\frac{1}{n} \log g(n)=m .
$$

Proof. By the argument in Theorem 5.2

$$
\left\langle\sigma_{\alpha} \sigma_{0}\right\rangle \leqq e^{-m \varrho(\alpha)}
$$

from which one finds that

$$
g(n) \leqq\left[(2 n+1)^{d-1}+\sum_{k=n+1}^{\infty}(2 d)(2 k+1)^{d-1} e^{-m(k-n)}\right] e^{-m n}
$$

which implies $\overline{\lim }\left(-\frac{1}{n} \log g(n)\right) \leqq m$. Since $g(n) \geqq f(n)$, the $\underline{\lim }$ is at most $m$ so (5.5) is proven. 
Remark. When reflection positivity fails, one should define $m$ by (5.5) if one wants the two theorems below to hold.

Theorem 5.5. If $n, k \geqq 0$, then

$$
g(n+k) \leqq g(n) g(k) \text {. }
$$

In particular

$$
g(n) \geqq e^{-m n}
$$

Proof. If $\beta_{1}=n+k$, then

$$
\left\langle\sigma_{0} \sigma_{\beta}\right\rangle \leqq \sum_{\alpha_{1}=n}\left\langle\sigma_{0} \sigma_{\alpha}\right\rangle\left\langle\sigma_{\alpha} \sigma_{\beta}\right\rangle
$$

by Theorem 2.1. Summing over all $\beta$ with $\beta_{1}=n+k$, we obtain (5.6). Thus $\ln g(n)$ is subadditive so

$$
\lim \left(\frac{1}{n} \ln g(n)\right)=\inf \left(\frac{1}{n} \ln g(n)\right)
$$

which is (5.7).

Theorem 5.6. Above the critical temperature, the susceptibility $\chi$ and the mass $m$ are related by:

$$
\chi \geqq \operatorname{coth}\left(\frac{m}{2}\right)
$$

Proof. $\chi=\sum_{n=-\infty}^{\infty} g(n)$ so that (5.8) is obtained by just summing (5.7).

Remarks. 1. Thus $\chi$ diverges at the critical point, a result obtained already by Glimm and Jaffe [14].

2. (5.8) implies the bound $\gamma \geqq v$ on critical exponents.

In $[14,15]$ Glimm and Jaffe defined a quantity $Z$ as the weight of the pole at $k=m$ in a certain spectral weight function. They proved $Z \neq 0$ for a.e. but did not obtain an explicit bound. (5.7) immediately implies our final results.

Theorem 5.7. The $Z$ of $[14,15]$ obeys $Z \geqq \sinh (m)$ for any nearest neighbor Ising model.

\section{References}

1. Agmon, S.: Hebrew University preprint; paper in preparation

2. Aizenman, M., Simon, B.: Commun. Math. Phys. 77, 137-143 (1980)

3. Aizenman, M., Simon, B.: Phys. Lett. (to appear)

4. Bricmont, J.: J. Stat. Phys. 17, 289 (1977)

5. Deift, P., Hunziker, W., Simon, B., Vock, E.: Commun. Math. Phys. 64, 7 (1970)

6. Dreisler, W., Landau, L., Fernando-Perez, J. : J. Stat. Phys. 20, 123 (1979)

7. Dobrushin, R.L.: Talks at Estrogom and Triblisi conferences

8. Dunlop, F.: J. Stat. Phys. (to appear)

9. Ellis, R., Monroe, J., Newman, C.: Commun. Math. Phys. 46, 167 (1976) 
10. Fisher, M.: Phys. Rev. 162, 480 (1967)

11. Fröhlich, J., Israel, R., Lieb, E., Simon, B.: Commun. Math. Phys. 62, 1 (1978)

12. Fröhlich, J., Spencer, T.: In preparation

13. Ginibre, J.: Commun. Math. Phys. 16, 310 (1970)

14. Glimm, J., Jaffe, A.: Commun. Math. Phys. 51, 1 (1976)

15. Glimm, J., Jaffe, A.: Commun. Math. Phys. 52, 263 (1977)

16. Glimm, J., Jaffe, A., Spencer, T.: Ann. Math. 100, 585 (1974)

17. Glimm, J., Jaffe, A., Spencer, T.: Constructive quantum field theory (eds. G. Yelo, A.S. Wightman, pp. 199-242. Berlin, Heidelberg, New York: Springer 1973

18. Griffiths, R.: J. Math. Phys. (N.Y.) 8, 478 (1967)

19. Griffiths, R.: J. Math. Phys. 8, 484 (1967)

20. Griffiths, R.: Commun. Math. Phys. 6, 121 (1967)

21. Griffiths, R.: J. Math. Phys. (N.Y.) 10, 1559 (1969)

22. Griffiths, R., Hurst, C., Sherman, S.: J. Math. Phys. (N.Y.) 11, 790 (1970)

23. Gross, L.: Commun. Math. Phys. (to appear)

24. Guerra, F., Rosen, L., Simon, B.: Ann. Math. 101, 111 (1975)

25. Kelley, D., Sherman, S. : J. Math. Phys. (N.Y.) 9, 466 (1968)

26. Shugard, W., Weeks, J., Gilmer, G. : Bell Labs Preprint

27. Krinsky, S., Emery, V.: Phys. Lett. 50A, 235 (1974)

28. Lebowitz, J.: Commun. Math. Phys. 35, 87 (1974)

29. Lebowitz, J.: Lecture Notes in Physics, Vol. 39, p. 370

30. Lieb, E.: Commun. Math. Phys. 77, 127-135 (1980)

31. McBryan, O., Rosen, J.: Commun. Math. Phys. 51, 97 (1967)

32. Newman, C.: Z. Wahrsch. 33, 75 (1975)

33. Reed, M., Simon, B.: Methods of modern mathematical physics. IV. Analysis of operators. London, New York: Academic Press 1978

34. Sylvester, G.: Harvard university thesis

35. Thompson, C.: Commun. Math. Phys. 24, 61 (1971)

36. Wells, D.: Indiana university preprint

Communicated by A. Jaffe

Received March 21, 1980

Note added in proof. In a series of papers, P. W. Kasteleyn and R. J. Boel [see Phys. Lett. 70A, 220 (1979), Commun. Math. Phys. 66, 167 (1979), and references therein] have found necessary and sufficient conditions for a class of inequalities to hold for arbitrary spin half Ising ferromagnets with a given set of "bonds" (basic interactions). The inequalities in Sect. 2 of the present paper are in the class considered by these authors and thus they are special cases of their inequalities and can be proven by their methods. Kasteleyn-Boel do not give applications of their inequalities. 